\title{
Die Thoraxdrainage
}

Michael Klopp, Hans Hoffmann, Hendrik Dienemann

Eine rasch angelegte Thoraxdrainage kann Leben retten - deshalb sollten nicht nur Chirurgen, sondern auch Notärzte und Intensivmediziner den Eingriff sicher beherrschen. Hier erfahren Sie, wann eine Thoraxdrainage indiziert ist und wie sie Schritt für Schritt durchgeführt wird.

\section{Indikationen}

Allgemeines | Bei jedem symptomatischen Pneumothorax ist die rasche Einlage einer Drainage die initiale Therapie. Je ausgeprägter die klinische Symptomatik ist, desto eher kann man auf diagnostische Schritte verzichten. Eine Ausnahme beim primären (idiopathischen) Spontanpneumothorax (PSP) ist der asymptomatische partielle PSP (Mantelpneumothorax, mit Pleuraspalt $<1$ Querfinger). Bei diesem kann man zunächst unter klinischer Kontrolle abwarten.

Notfallsituationen | Wenn bei einem symptomatischen Spannungspneumothorax eine reguläre Drainageneinlage nicht sofort möglich ist, kann man durch Punktion interkostal mit einer großlumigen Verweilkanüle den Pneumothorax temporär entlasten.

Kommt es zu einem Pneumothorax unter mechanischer Ventilation, können Patienten rasch einen Spannungspneumothorax entwickeln. Deshalb besteht in diesem Fall nahezu immer die Indikation zu sofortiger Drainageneinlage.

Hämatothorax | Ein Hämatothorax bedarf einer Pleuradrainage, um

- die Blutungsaktivität zu ermitteln,

- die Lungenwiederausdehnung zu ermöglichen

- und somit eine Fesselung der Lunge und ein Spätempyem zu vermeiden.

Kontrovers wird diskutiert, ob eine Drainage bei parapneumonischen Flüssigkeitsansammlungen nötig ist. Allgemein akzeptiert ist die Indikation zur Drainageneinlage, wenn ein Pleuraempyem im Stadium II (ATS-Klassifikation 1962) vorliegt. Eine Drainageneinlage beim Pleuraempyem im Stadium I kann bei Resterguss oder nicht vollständig entfalteter Lunge die zügige komplette Entleerung der Pleurahöhle mit Reexpansion der atelektatischen Lungenanteile unterstützen. Ein Pleuraerguss auf dem Boden einer pleuralen Tumorzellaussaat bedarf einer Drainage, sofern er symptomatisch ist oder eine Pleurodese erwogen wird. Dasselbe gilt für einen Chylothorax jeglicher Ursache.

\section{Kontraindikationen}

Absolute Kontraindikationen bestehen nicht. Eine relative Kontraindikation ist eine Blutungsdiathese oder Antikoagulation. Besondere Umstände ergeben sich gegebenenfalls beim Vorhandensein

- pleuraler Adhäsionen,

- abgekapselten Flüssigkeitsansammlungen,

- Riesenbullae (die als Pneumothorax fehlgedeutet werden können) und

- eines Verdachts auf Zwerchfellruptur bei Traumapatienten.

Unter diesen Umständen sollten bildgebende Verfahren (CT, Ultraschall, Durchleuchtung) eingesetzt und Operationsbereitschaft gegeben sein.

\section{Patientenaufklärung}

Der Patient muss über Indikation, Technik und unmittelbare Auswirkungen auf das Befinden aufgeklärt werden. Insbesondere die Lokalanästhesie sowie die Möglichkeit einer zusätzlichen Sedierung sollten Sie erwähnen.

Mögliche Komplikationen | Weiterhin sollte der Patient das Wichtigste über folgende Komplikationsmöglichkeiten erfahren:

- Drainagenfehllage

- Organverletzungen mit Blutung, bronchopleuraler Fistel und Weichteilemphysem

- Pleuraempyem

- Reexpansion (-södem) der Lunge mit ev. Hustenreiz, Schulter- und Thoraxschmerz sowie vagalen Reaktionen

- Läsion der Interkostalgefäße/-nerven und des Rippenperiosts mit Nachblutungen, Schmerzen und Interkostalneuralgien

- Folgeoperationen

\section{Vorbereitung}

Vor dem Start I Die Drainage dürfen Sie nur nach sorgfältiger klinischer Untersuchung und in Kenntnis eines aktuellen Röntgenbildes, Thorax-CT oder Ultraschallbefundes anlegen. Eine Ausnahme ist der dringende klinische Verdacht 
Abb. 1 Die Ausstattung zur Pleuradrainageneinlage: (A) Desinfektionsmittel, (B) Lokalanästhesie und Punktionsnadel, (C) sterile Abdeckung, Handschuhe und Kittel, (D) Skalpell und Pinzetten, (E) Präparierschere, (F) Kornzange und Pleuradrainage (28 Charrière), (G) Nadelhalter, (H) Fadenschere und Nahtmaterial (Surgilon 1 zur Drainagenannaht, Vicryl 3-0 für eventuell erforderliche Subkutannaht, Seralon $3 / 0$ als Hautnaht). auf einen progredienten Spannungspneumothorax (mit Blutdruck-, Sättigungsabfall, Tachypnoe, Einflussstauung). Hier können Sie meist nicht auf ein Röntgenbild warten.

Material | Die Drainageneinlage erfolgt unter sterilen Kautelen. $>$ Abb. 1 zeigt die verwendeten Materialien.

Lage des Patienten | Bei nicht-gekammerten Prozessen wird die Drainage am liegenden Patienten angelegt bei mind. $90^{\circ}$ abduziertem Arm, der bequem gelagert oder gehalten wird. Die betroffenen Seite des Patienten wird leicht angehoben.

Zugangsort finden | Für die Einlage wählt man den 3.-5. Interkostalraum in der vorderen oder mittleren Axillarlinie. Als Orientierungshilfe dient beim Mann die Mamille (entspricht der 4. Rippe) oder die Submammarfalte bei der Frau (4. Interkostalraum bis 5. Rippe). Als Eintrittstelle dient das sogenannte muskelfreie Dreieck zwischen dem Seitenrand des $M$. pectoralis major und dem Vorderrand des M. latissimus dorsi. Bei abgekapselten Prozessen ist eine gezielte Einlage nötig, u.U. unter direkter Ultraschall- oder CT-Kontrolle.

Gelegentlich wird der 2. Interkostalraum in der Medioklavikularlinie („Monaldi-Position”) für die Pleuradrainageneinlage empfohlen. Vorteil ist die zuverlässige Orientierung am liegenden Patienten (Ansatz der 2. Rippe in Höhe des leicht identifizierbaren Angulus sterni). Nachteile sind jedoch:

- Risiko einer Perforation des Musculus pectoralis major mit entsprechendem Blutungsrisiko

- die Schwierigkeit, die Drainage gezielt apikal oder basal zu platzieren

- die Gefahr, dass die Drainage rechtwinklig abknickt

- das ungünstige kosmetische Ergebnis bei Narbenbildung

Noch ungünstiger ist der posteriore Zugang medial der Skapula, der ausschließlich bei dorsal lokalisierten Prozessen indiziert ist.

\section{Thoraxdrainage: So wird's gemacht}

Betäubung I Nachdem Sie die Haut desinfiziert und steril abgedeckt haben, folgt die Infiltration von 10-20 ml Lokalanästhetikum subkutan, perikostal und interkostal mit Anästhesie der parietalen Pleura ( $\triangle$ Abb. 2). Mit der gleichen Nadel durchstoßen Sie die Pleura parietalis. Wird dabei Luft oder Flüssigkeit aspiriert, haben Sie einen geeigneten Interkostalraum gefunden.

Eröffnung der Pleura | Führen Sie eine $2 \mathrm{~cm}$ lange Hautinzision durch und spreizen Sie Subkutis und Serratusmuskulatur ( Abb. 3). Eröffnen Sie vorsichtig die Interkostalmuskulatur und anschließend die parietale Pleura. Dabei können Sie sich am Oberrand der Rippe orientieren. Die Eröffnung geschieht entweder mittels einer kurzer Schere, die stumpf eingeführt und beim Zurückziehen leicht gespreizt wird, oder bei ausreichend weiten Interkostalräumen direkt mit dem sterilen Zeigefinger $($ Abb. 4). Meist entweicht die unter Druck stehende Luft erkennbar durch ein Zischen.

Abtasten | Tasten Sie den Pleuraum ab und überprüfen sie ihn auf mögliche Verwachsungen.

Einführen der Drainage | Führen Sie die Thoraxdrainage stumpf (ohne Trokar!) mittels gebogener Kornzange ( Abb. 5) über den vorbereiteten Gewebskanal nach dorsal-apikal (Pneumothorax) bzw. dorsal-kaudal bei Überwiegen des Pleuraergusses (Sero-Pneumothorax) ein. Die Drainagegrößen sollte mind. 20 Charrière beim Pneumothorax und 24-28 Charrière bei (zusätzlichem) Erguss bzw. Blut betragen. Bei korrekter Durchführung ist nur ein sehr geringer und gleichmäßiger Widerstand beim Einführen zu spüren. Ein federnder Widerstand deutet ein Anstoßen oder Abknicken der Drainage an.

Knickt die Drainage beim Einführen bereits über dem Hautniveau ab, so ist eine Via falsa außerhalb des knöchernen Thorax zu vermuten.

Wird eine lufthaltige Pleurahöhle drainiert, so beschlägt der Drainageschlauch mit jeder Exspiration. Dass das System durchgängig ist, erkennen Sie an atemsynchronen Schwankungen der Flüssigkeitssäule im Schlauchsystem oder des Flüssigkeitsspiegels im Auffangbehälter. Entleert sich atemsynchron Luft, sollten Sie überprüfen, ob das ableitenden System undicht ist. Ist dies nicht der Fall, könnte eine Parenchymfistel vorliegen.

Befestigung der Drainage | Nachdem Sie sich von der korrekten Lage und Durchgängigkeit des Systems überzeugt haben, fixieren Sie die Drainage mit kräftigem Faden mittels U-Naht ( Abb. 6) an der Haut (luft-, flüssigkeitsdicht). Die Annaht wird so geknüpft ( Abb. 7), dass sie bei Entfernen der Drainage als Zuziehfaden dient. Dazu legen Sie 


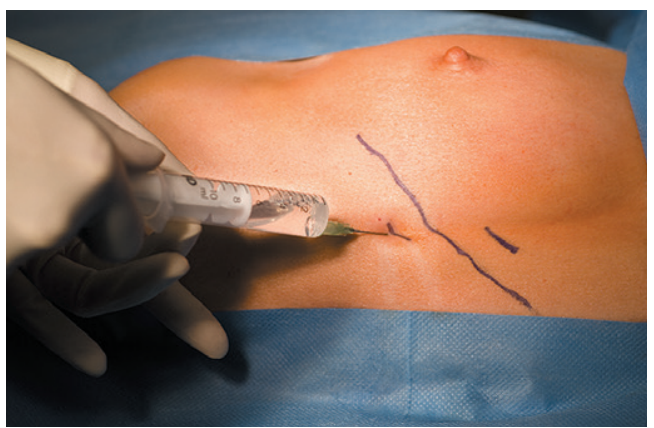

Abb. 2 Lokalanästhesie und Aspiration von Luft.

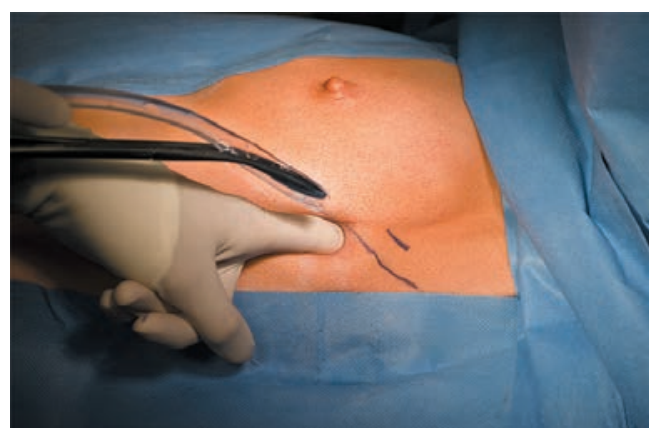

Abb. 4 Stumpfes Eingehen nach intrathorakal und digitale Austastung.

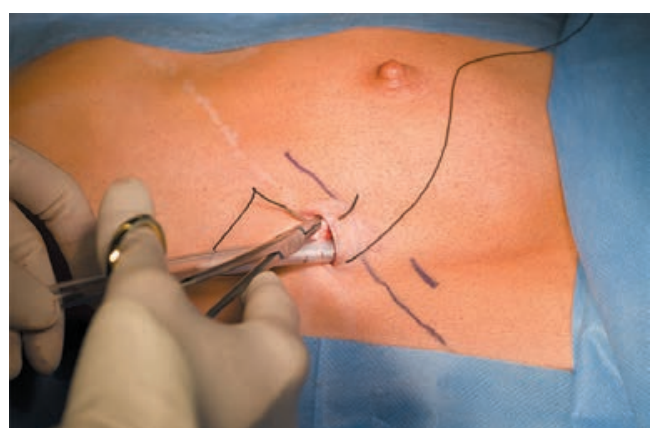

Abb. 6 Drainagennaht mit U-Naht.

einen Knoten nach der U-Naht straff vor und umwickeln die Drainage mehrfach und machen einen Doppelknoten. Es folgt die sterile Konnektion der Pleuradrainage mit einem Auffangbehälter nach dem Bülau-Prinzip. Ggf. können Sie ein Sog von $-15 \mathrm{~cm} \mathrm{H}_{2} \mathrm{O}$ an das Drainagesystem anschließen.

Röngen-Thorax | Ein anschließender RöntgenThorax in 2 Ebenen dokumentiert die korrekte Position der Drainage und die Reexpansion der Lunge.

Drainagesysteme I „Passive” Drainagesysteme erlauben die Ableitung von Gas oder Flüssigkeit während der Exspiration. Es empfiehlt sich das Unterwasserventil-Prinzip nach Bülau. Ein mit Wasser befüllter Behälter dient dabei als Sammelgefäß wie auch als Ventil ( Abb. 8). „Aktive” Drainagesysteme haben eine zusätzliche Unterdruckanlage am ableitenden Schenkel des Auffangsystems. Diese sind bei verzögerter Reexpansion der Lunge erforderlich. In der Regel ist ein Druck von -15 bis max. $-30 \mathrm{~cm} \mathrm{H}_{2} \mathrm{O}$ ausreichend. Entfaltet sich die Lunge nicht, kann die Sogtherapie kontraproduk-

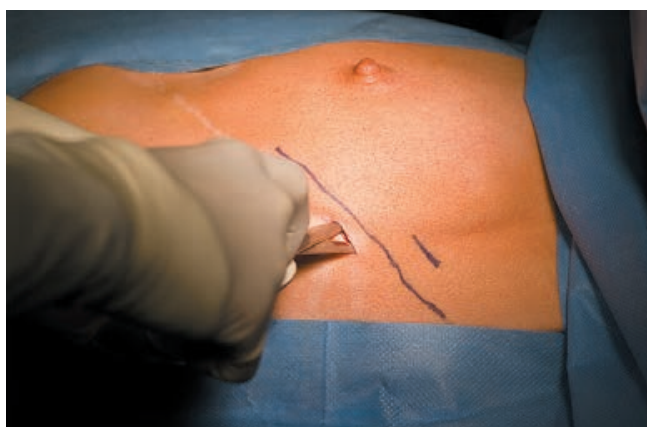

Abb. 3 Präparation mit der Schere (Aufspreizen).

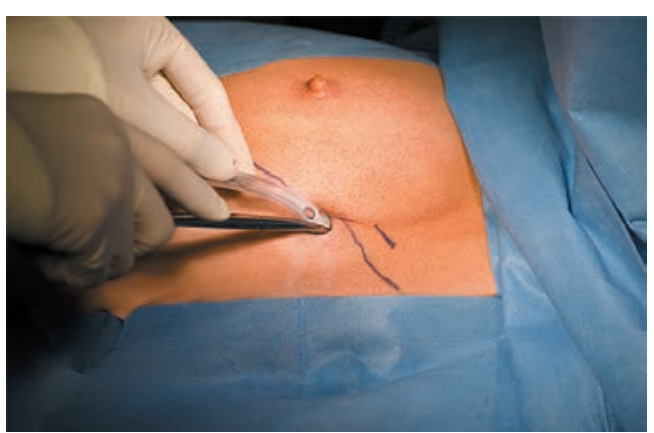

Abb. 5 Pleuradrainageneinlage nach dorsal-apikal.

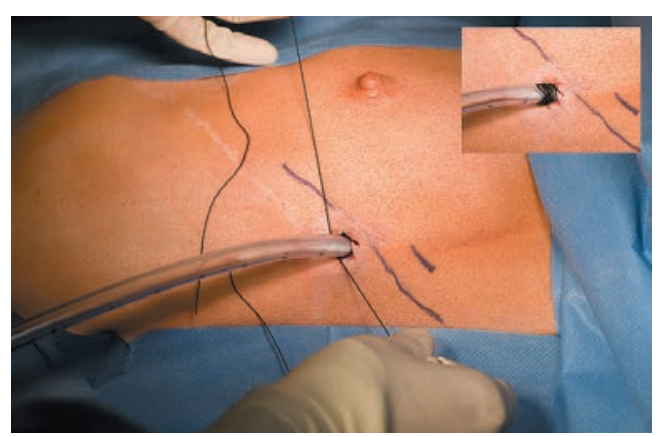

Abb. 7 Annaht des Drainageschlauchs.

tiv sein, da sie eine bestehende Parenchymfistel aufrechterhalten könnte.

\section{Entfernung der Drainage}

Zeitpunkt | Die Entfernung der Pleuradrainage ist indiziert, wenn

- eine vollständige Reexpansion der Lunge erreicht ist,

- Parenchymfisteln sistieren und

- der Verlust an Pleuraflüssigkeit über 24 Stunden unter 150-200 $\mathrm{ml}$ beträgt oder

- von einer Ausheilung des Empyems (negative Drainagenabstriche) ausgegangen werden kann.

Ausschlaggebend ist letztlich immer die individuelle Situation, insbesondere, wenn der Pleuraraum zusätzlich infiziert ist. Nach spätestens $24 \mathrm{~h}$ muss zur Kontrolle ein Röntgen-Thorax erfolgen.

Cave Eine Drainage, die „fistelt”, darf niemals abgeklemmt oder entfernt werden.

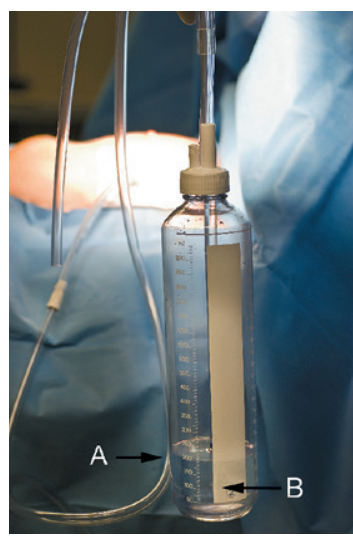

Abb. 8 Passives Drainagesystem (Unterwasserventil-Prinzip nach Bülau 1891). A=Befüllen der Flasche mit $200 \mathrm{ml}$ destilliertem Wasser; $B=$ Eintauchen des Drainageschlauches, um das Unterwasserventil zu aktivieren. 


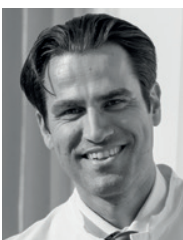

Dr. med. Michael Klopp ist Oberarzt an der Thoraxklinik des Universitätsklinikums Heidelberg.

michael.klopp@urz.uni-heidelberg.de

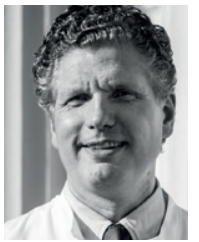

Prof. Dr. med. Hans Hoffmann ist leitender Oberarzt Chirurgie der Thoraxklinik am Universitätsklinikum Heidelberg.

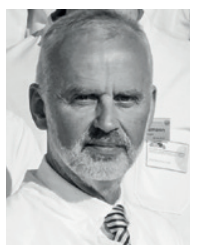

Univ. Prof. Dr. med.

Hendrik Dienemann ist ärztlicher Direktor und Medizinischer Geschäftsführer der Thoraxklinik und Chefarzt der Abteilung Chirurgie am Universitätsklinikum Heidelberg.

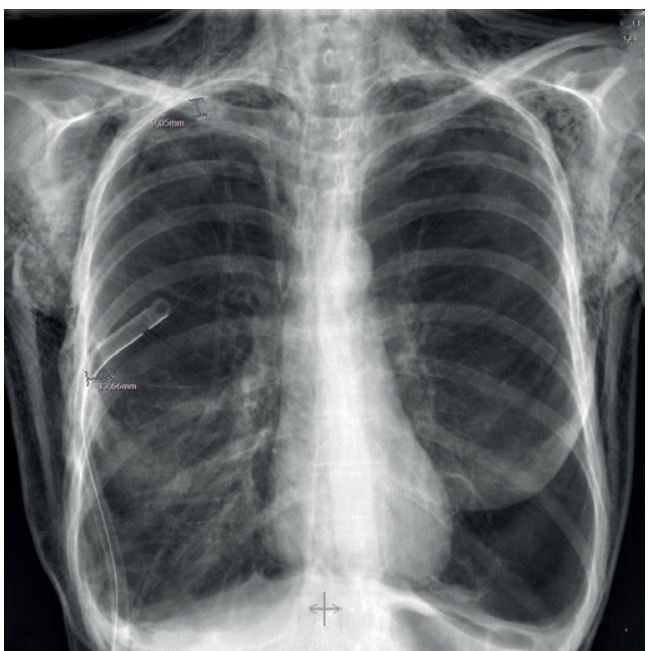

Abb. 9 Weichteilemphysem bei broncho-pleuraler Fistel.

\section{Probleme und Lösungen}

Fehllage I Ein zu tiefes Einführen der Drainage verursacht bei Druck auf die parietale Pleura Brust- oder Schulterschmerz. Die zu kurz eingelegte oder unzureichend fixierte Drainage induziert bei Pneumothorax ein Weichteilemphysem.

Weichteilemphysem | Bei einem Weichteilemphysem ( $\bullet$ Abb. 9) müssen Sie die Drainagenlage und die Dichtigkeit und Durchgängigkeit des ableitenden Systems überprüfen. Auch große broncho- oder alveolo-pleurale Fisteln können ein Weichteilemphysem verursachen, wenn das Drainagesystem zu klein dimensioniert ist. In diesen Fällen sollten Sie ein aktives Drainagesystem wählen. Entleert sich blutiger Schaum, liegt häufig eine direkte Parenchymverletzung mit konsekutiver Blutung und Luftleck vor. Meistens erfordert dies eine zweite Drainage und gegebenenfalls die operative Revision.

Organverletzungen | Das Risiko für Organverletzungen lässt sich minimieren, indem man auf Trokardrainagen verzichtet. Besonders exponiert sind Lunge, Milz, Leber und Magen sowie das Zwerchfell selbst. Problematisch ist meist, dass ein Zwerchfellhochstand nicht erkannt wird, z. B. bei

- Phrenikusparese,

- adipöse Patienten in liegender Position,

- nach Pneumonektomie.

Blutung I Geringfügige Blutungen kommen meist spontan zum Stillstand und verfärben nur anfänglich das Pleuraprodukt. Starke Blutungen können nach Verletzung der Interkostalarterie auftreten. Sie lassen sich jedoch vermeiden, wenn man sich bei Drainageneinlage am Oberrand einer Rippe orientiert. Profuse Blutungen deuten auf eine direkte Gefäßverletzung (Hohlvenen, Aorta, Pulmonalarterie, Herz) oder einen vorhandenen Hämothorax nach Trauma oder Voroperation hin.

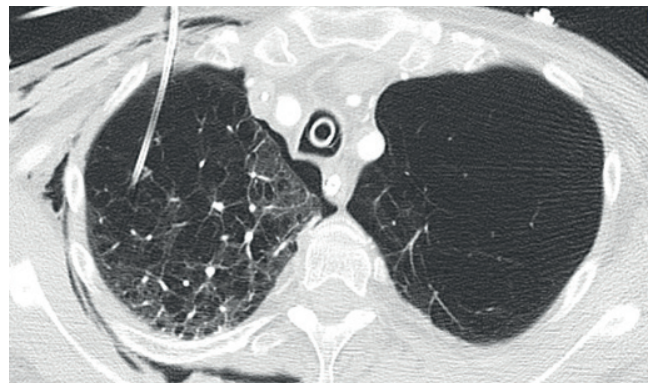

Abb. 10 Lungenperforation bei bullösem Emphysem, nach Trokar-Drainageneinlage in Monaldi Position.

Diese seltenen Komplikationen resultieren stets aus der unsachgemäßen Einlage von Trokardrainagen. Auch tangentiale Zwerchfellverletzungen können einen bedrohlichen Blutverlust verursachen. Kommt es zu einem akuten Abdomen oder einem hämorrhagischen Schock, können transdiaphragmale Verletzungen abdominaler Organe vorliegen.

Pleuraempyem | Bei primär sterilem Pleurainhalt und korrekter Drainageneinlage ist bei einer vollständigen Reexpansion der Lunge ein Pleuraempyem kaum zu befürchten. Liegen keine Risikofaktoren vor, ist die prophylaktische Antibiotikagabe abzulehnen. Eine über mehrere Tage bestehende Parenchymfistel begünstigt die Entstehung eines Pleuraempyems, weshalb eine frühzeitige operative Revision erwogen werden muss.

Reexpansionsödem | Das unilaterale Reexpansionsödem der Lunge ist eine seltene, jedoch potentiell letale Komplikation. Gefährdet sind Patienten, bei denen nach längerfristiger ( $>3$ Tage) Totalatelektase die Lunge rasch reexpandiert. Die Klinik ist gekennzeichnet durch starken Hustenreiz, Produktion von hellrotem Sputum, Tachypnoe und Tachykardie. Röntgenologisch findet sich ein einseitiges Lungenödem, das bis zu 24 Stunden nach Reexpansion auftreten kann. Als prophylaktische Maßnahme ist das intermittierende Abklemmen der Drainage empfohlen, um die Reexpansionsphase auf etwa $24 \mathrm{~h}$ auszudehnen.

\section{Konsequenz für Klinik und Praxis}

- Vor der Drainageneinlage Indikation und OP-Seite mittels Röntgen-Thorax sichern.

- Bevorzugte Inzisionsstelle ist der 4./5. Interkostalraum in der mittleren Axillarlinie.

- Nach Durchtrennung der Pleura wird der Pleuraraum mit dem Finger stumpf ausgetastet, um Verwachsungen auszuschließen und sich der intrathorakalen Lage zu vergewissern.

- Die Drainage darf erst nach „Fistelausschluss” und bei radiologisch dokumentierter Entfaltung der Lunge entfernt werden.

Vollständiges Literaturverzeichnis unter: http://dx.doi.org/10.1055/s-0041-100725 


\section{Literatur}

1 Henry M, Arnold T, Harvey J. BTS guidelines for the management of spontaneous pneumothorax. Thorax 2003; 58 Suppl 2: ii39-52

2 Klopp M, Dienemann H, Hoffmann H. Behandlung des Pneumothorax. Chirurg 2007; 78 (7): 655-68

3 Klopp M, Pfannschmidt J, Dienemann H. Behandlung des Pleuraempyems. Chirurg 2008; 79 (1): 83-96 